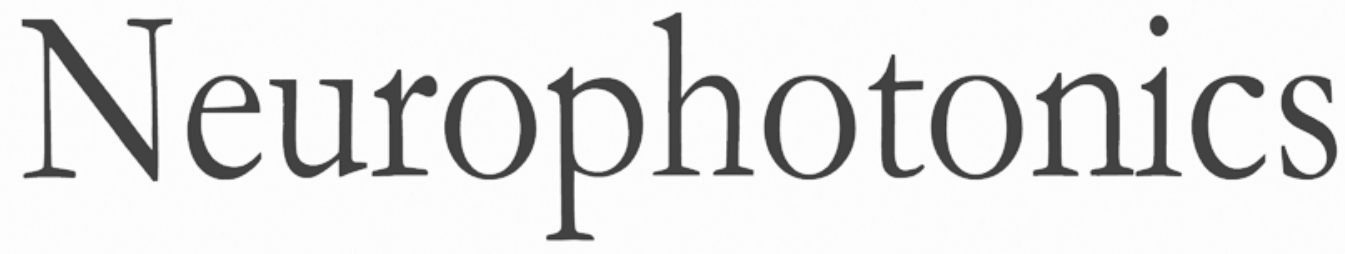

\title{
Transcranial photobiomodulation with 1064-nm laser modulates brain electroencephalogram rhythms
}

Xinlong Wang

Jacek P. Dmochowski

Li Zeng

Elisa Kallioniemi

Mustafa Husain

F. Gonzalez-Lima

Hanli Liu 


\title{
Transcranial photobiomodulation with 1064-nm laser modulates brain electroencephalogram rhythms
}

\author{
Xinlong Wang, ${ }^{a}$ Jacek P. Dmochowski, ${ }^{b}$ Li Zeng, ${ }^{c}$ Elisa Kallioniemi, ${ }^{d}$ Mustafa Husain, ${ }^{d}$ F. Gonzalez-Lima, ${ }^{e}$ and \\ Hanli Liu ${ }^{\mathrm{a}, \star}$ \\ aUniversity of Texas at Arlington, Department of Bioengineering, Arlington, Texas, United States \\ ${ }^{b}$ City College of New York, Department of Biomedical Engineering, New York, United States \\ ${ }^{C}$ Texas A\&M University, Department of Industrial and Systems Engineering, College Station, Texas, United States \\ dUniversity of Texas Southwestern Medical Center at Dallas, Department of Psychiatry, Dallas, Texas, United States \\ eUniversity of Texas at Austin, Department of Psychology and Institute for Neuroscience, Austin, Texas, United States
}

\begin{abstract}
Noninvasive transcranial photobiomodulation (tPBM) with a 1064-nm laser has been reported to improve human performance on cognitive tasks as well as locally upregulate cerebral oxygen metabolism and hemodynamics. However, it is unknown whether 1064-nm tPBM also modulates electrophysiology, and specifically neural oscillations, in the human brain. The hypothesis guiding our study is that applying 1064-nm tPBM of the right prefrontal cortex enhances neurophysiological rhythms at specific frequency bands in the human brain under resting conditions. To test this hypothesis, we recorded the 64-channel scalp electroencephalogram (EEG) before, during, and after the application of $11 \mathrm{~min}$ of 4-cm-diameter tPBM (CW 1064-nm laser with $162 \mathrm{~mW} / \mathrm{cm}^{2}$ and $\left.107 \mathrm{~J} / \mathrm{cm}^{2}\right)$ to the right forehead of human subjects $(n=20)$ using a within-subject, sham-controlled design. Time-resolved scalp topographies of EEG power at five frequency bands were computed to examine the tPBM-induced EEG power changes across the scalp. The results show time-dependent, significant increases of EEG spectral powers at the alpha (8 to $13 \mathrm{~Hz}$ ) and beta (13 to $30 \mathrm{~Hz})$ bands at broad scalp regions, exhibiting a front-to-back pattern. The findings provide the first sham-controlled topographic mapping that tPBM increases the strength of electrophysiological oscillations (alpha and beta bands) while also shedding light on the mechanisms of tPBM in the human brain. $\odot$ The Authors. Published by SPIE under a Creative Commons Attribution 4.0 Unported License. Distribution or reproduction of this work in whole or in part requires full attribution of the original publication, including its DOI. [DOI: 10.1117/1.NPh.6.2.025013]
\end{abstract}

Keywords: transcranial photobiomodulation; electroencephalogram; neurophysiological rhythms; low-level light therapy; infrared laser.

Paper 19004R received Jan. 9, 2019; accepted for publication May 22, 2019; published online Jun. 13, 2019.

\section{Introduction}

Photobiomodulation (PBM) is a process that uses light, with nondestructive and nonthermal delivery techniques, to modulate mitochondrial respiration and cellular functions in many cell types, including neurons. ${ }^{1-4}$ PBM has also been called low-level laser (light) therapy (LLLT), when its goal is to serve as a therapy for a variety of medical conditions, such as for pain relief and wound healing. ${ }^{5,6}$ When PBM is aimed at the human head, the term transcranial photobiomodulation (tPBM) has been used to emphasize that the target modulated by light is the human cerebral cortex, up to $\sim 3 \mathrm{~cm}$ below the human scalp, ${ }^{7}$ with the goal of enhancing cerebral oxygenation and cognitive functioning. ${ }^{8-10}$ In general, most research studies or clinical applications with tPBM have utilized either lasers or light-emitting diodes (LEDs) in the red (620 to $680 \mathrm{~nm}$ ) or near-infrared (800 to $980 \mathrm{~nm}$ ) spectral range. ${ }^{11}$ LEDs with a bandwidth of 1064 to $1080 \mathrm{~nm}$ were applied to neuromodulate the human brain of a small group of dementia patients (six active, three controls, and two dropouts) and to demonstrate the feasibility of tPBM being able to improve the patients' executive functioning. ${ }^{12}$ This study also reports a trend of tPBM-induced improvement in electroencephalogram (EEG) amplitude and connectivity. Furthermore, a 1064-nm infrared laser has been utilized to apply tPBM and is reported to improve cognitive

*Address all correspondence to Hanli Liu, E-mail: hanli@uta.edu performance on various tasks in sham-controlled studies with more than 300 human subjects. ${ }^{13-16}$ More recently, we have demonstrated that tPBM by 1064-nm laser induced significant changes in cerebral blood oxygenation as measured with functional near-infrared spectroscopy. ${ }^{17,18}$ These changes are caused by neuron-photon interaction that induces increases in the concentration of oxidized cytochrome-c-oxidase (CCO), ${ }^{18}$ the enzyme at the terminal stage of the mitochondrial respiratory chain. $^{19}$

The previous in vivo hemodynamic and metabolic tPBM studies with 1064-nm laser in humans provide insights into the physiological changes evoked by stimulation with a 1064-nm infrared laser. ${ }^{18,19}$ The purported mechanism of tPBM in improving human cognitive function is a neuron-photon interaction initiated by $\mathrm{CCO}$ photo-oxidation. ${ }^{8,18}$ In brief, incident photons cause CCO to lose electrons (i.e., to become photooxidized) since $\mathrm{CCO}$ is the main photon acceptor in cells. When CCO becomes oxidized, it has a conformation with more affinity to catalyze the reduction of oxygen to water in cellular respiration. The increment of oxidized CCO concentration, therefore, accelerates ATP production via oxidative phosphorylation by consuming more oxygen molecules. ${ }^{8,18,20}$ The faster consumption rate of oxygen molecules triggers an increase in blood flow and thus hemodynamic oxygenation from nearby blood vessels. The increased ATP in neurons is then utilized as an energy supply to carry out a variety of cellular activities and biochemical reactions. ${ }^{8,20}$ In the meantime, it is possible that 
tPBM light can be absorbed by and/or can interact with a variety of cells and organelles in human tissue due to the high complexity of biological tissue. For example, PBM is able to modulate gene expression and calcium ion channel activities, as reported in preclinical studies. ${ }^{21}$

Since the most energy-demanding cellular activity in neurons involves maintaining membrane electrical potentials, ${ }^{22}$ the tPBM-induced increase in oxidized CCO and ATP concentrations in neurons may potentially lead to electrophysiological alterations. Moreover, according to the findings in our previous studies, local hemodynamic and metabolic responses are reported as responses to $\mathrm{TPBM}$ on the right prefrontal cortex. However, it is yet unknown if the tPBM induces distal/global electrophysiological responses due to neuronal connectivity. To date, only a couple of in vivo studies have investigated the electrophysiological changes driven by tPBM. ${ }^{16,23}$ The primary goal of this study was to determine whether TPBM modulates the amplitude of human cortical rhythms as measured by the scalp EEG before, during, and after tPBM.

The scalp EEG is a time-resolved measure of the aggregate synaptic activity across millions of cells in the underlying cerebral cortex. EEG has been often combined with brain stimulation [e.g., transcranial direct current stimulation and transcranial magnetic stimulation (TMS)]) to study the mechanisms of neuromodulation techniques. ${ }^{24-26}$ Modulation in the activity of alpha (8 to $13 \mathrm{~Hz})$, beta (13 to $30 \mathrm{~Hz}$ ), and gamma (30 to $70 \mathrm{~Hz})$ rhythms has long been associated with cognitive function and brain states. ${ }^{27-29}$ Moreover, a variety of forms of tPBM (using either LEDs at wavelengths between 800 and $850 \mathrm{~nm}$ or a laser at $1064 \mathrm{~nm}$ ) have been reported to enhance human cognitive functions, such as working memory, sustained attention, category learning, and executive skills. ${ }^{8,10,11,13-16,30}$

To the best of our knowledge, few studies have reported EEG spectral power changes in response to tPBM (except for two preliminary studies from our own groups ${ }^{16,23}$ ). Therefore, in this study we have focused on two major research questions. First, does tPBM significantly modulate human cortical rhythms as compared to sham stimulation? If so, what frequency bands are responsive to $\mathrm{PBM}$ ? Second, what is the spatial distribution of tPBM-induced alterations in oscillatory power? Specifically, we hypothesize that $\mathrm{PBM}$ of the right prefrontal cortex enhances neurophysiological rhythms at specific frequency bands in the human brain under resting conditions.

To test our hypothesis, we recorded scalp EEG before, during, and after the application of 1064-nm infrared laser on the right frontal forehead in $n=20$ subjects using a within-subjects, sham-controlled design. By the end of this study, the hypothesis was confirmed by our observations that $\mathrm{PBM}$ gave rise to significant increases in the spectral power strength of electrophysiological oscillations at alpha and beta bands across anterior to posterior regions in the human brain, revealing electrophysiological mechanisms of action of tPBM on the human brain.

\section{Materials and Methods}

\subsection{Participants}

A total of 20 healthy human participants with an average $( \pm$ SD) age of $26.8 \pm 8.8$ years were recruited from the local community of the University of Texas at Arlington, Texas. Specifically, 7 females $(24.0 \pm 4.1$ years of age) and 13 males $(28.2 \pm 10.3$ years of age) participated in the study; the two gender groups had no significant age difference (with a two-tailed $t$-test, $p>0.2$ ). The exclusion criteria included: (1) previous diagnosis with a psychiatric disorder, (2) history of neurological disease, (3) history of severe brain injury, (4) history of violent behavior, (5) prior institutionalization/imprisonment, (6) current intake of any psychotropic medicine or drug, (7) previous diagnosis with diabetes, (8) history of smoking, (9) excessive alcohol consumption, or (10) pregnancy. Exclusion of diabetic patients was required by the manufacturer of the laser (Cell Gen Therapeutics LLC, Dallas, Texas); lack of prior psychiatric diagnosis was ascertained by self-report. The study protocol was approved by the Institutional Review Board (IRB) at the University of Texas in Arlington. Informed consent was obtained prior to all experiments.

\subsection{Experimental Setup for Simultaneous EEG-tPBM Measurements}

We employed an FDA-cleared, 1064-nm, continuous-wave (CW) laser (Model CG-5000 Laser, Cell Gen Therapeutics LLC, Dallas, Texas) used in previous studies. ${ }^{13-18,30}$ Tables $1-3$ list the tPBM parameters used in this study following the recommended format given in Ref. 31. The laser's aperture delivered a wellcollimated beam with an area of $13.6 \mathrm{~cm}^{2}$. To avoid thermal sensation for a more truthful sham-controlled study, we decreased the laser power to $2.2 \mathrm{~W}$ from $3.4 \mathrm{~W}$ used in previous studies. ${ }^{13-18,30}$ Indeed, all participants reported no thermal sensation. This laser power of $2.2 \mathrm{~W}$ resulted in a power density of $0.162 \mathrm{~W} / \mathrm{cm}^{2}$, energy density of $\sim 107 \mathrm{~J} / \mathrm{cm}^{2}$, and a total energy dose of $1452 \mathrm{~J}$ over the 11-min tPBM duration $(2.2 \mathrm{~W} \times 660 \mathrm{~s}=1452 \mathrm{~J})$. Note that the optical energy density (or fluence) received on the cortical region would be only $1 \%$ to $2 \%$ of that delivered on the scalp by the laser aperture, based on recent literature. ${ }^{7,32-34}$ For sham stimulation, the laser power output was set to $0.1 \mathrm{~W}$, and a black cap was also placed in front of the aperture to further block the laser. The corresponding power density under the sham stimulation was further tested to be $0 \mathrm{~mW} / \mathrm{cm}^{2}$ by a sensitive power meter (Model $843-\mathrm{R}$, Newport Corporation, Massachusetts) to ensure complete blockage of laser light.

The experimental setup of the simultaneous EEG-tPBM measurements is shown in Figs. 1(a) and 1(b). The EEG data were collected using a 64-channel EEG instrument (ActiveTwo, Biosemi, the Netherlands). Channel $48, \mathrm{Cz}$, was used as the

Table 1 Device information.

\begin{tabular}{|c|c|}
\hline Device information & Specifications \\
\hline Manufacturer & $\begin{array}{l}\text { Cell Gen Therapeutics LLC, } \\
\text { Dallas, Texas }\end{array}$ \\
\hline Model identifier & CG-5000 Laser \\
\hline Year produced & 2017 \\
\hline Number of emitters & 1 \\
\hline Emitter type & Laser \\
\hline Spatial distribution of emitters & $\begin{array}{l}\text { Single emitter (followed by } \\
\text { collimation) }\end{array}$ \\
\hline Beam delivery system & Handheld probe \\
\hline
\end{tabular}


Table 2 Irradiation parameters.

\begin{tabular}{lc} 
Irradiation parameters & Value \\
\hline Center wavelength $(\mathrm{nm})$ & 1064 \\
Spectral bandwidth $(\mathrm{nm})$ & $5(\mathrm{FWHM})$ \\
Operating model & $\mathrm{CW}$ \\
Frequency $(\mathrm{Hz})$ & $\mathrm{N} / \mathrm{A}$ \\
Pulse on duration $(\mathrm{s})$ & $\mathrm{N} / \mathrm{A}$ \\
Pulse off duration $(\mathrm{s})$ or & $\mathrm{N} / \mathrm{A}$ \\
Duty cycle $(\%)$ & $\mathrm{N} / \mathrm{A}$ \\
Energy per pulse $(\mathrm{J})$ & $\mathrm{N} / \mathrm{A}$ \\
Peak radiant power $(\mathrm{mW})$ & $\mathrm{N} / \mathrm{A}$ \\
Average radiant power $(\mathrm{mW})$ & 2200 \\
Polarization & No (after fiber transmission) \\
Aperture diameter $(\mathrm{cm})$ & 4 \\
Irradiance at aperture $\left(\mathrm{mW} / \mathrm{cm}^{2}\right)$ & 162 \\
Beam divergence $(\mathrm{rad}$ or deg) & Near zero (collimated beam) \\
Beam shape & Circular \\
Beam profile & Top Hat \\
\hline
\end{tabular}

Table 3 Treatment parameters.

\begin{tabular}{lc} 
Treatment parameters & Value \\
\hline Beam spot size at target $\left(\mathrm{cm}^{2}\right)$ & 13.6 \\
Irradiance at target $\left(\mathrm{mW} / \mathrm{cm}^{2}\right)$ & 162 \\
Exposure duration $(\mathrm{s})$ & 660 \\
Radiant exposure $\left(\mathrm{J} / \mathrm{cm}^{2}\right)$ & 107 \\
Radiant energy $(\mathrm{J})$ & 1455 \\
Number of points irradiated & 1 \\
Area irradiated $\left.(\mathrm{cm})^{2}\right)$ & 13.6 \\
Application technique & Interstitial fiber optic \\
Number and frequency of & One session; \\
treatment sessions & delivered in 11 min \\
Total radiant energy $(\mathrm{J})$ & 1455 \\
\hline
\end{tabular}

reference. The tPBM was applied by noncontact delivery to the right forehead of each subject at a frontal site (near the Fp2 location of the international 10-10 system); the same laser light delivery location demonstrated to enhance cognitive performance and local oxygen metabolism in our previous studies. ${ }^{14-16,18}$ Laser protection goggles with extra black-tape rims were worn by the subject and the experimental operator throughout the entire experiment, before, during, and after tPBM. To prevent sleepiness or drowsiness, the subjects kept their eyes open during the EEG-tPBM measurements after the laser aperture was well positioned on their forehead. Each subject wore an EEG cap with 64 electrodes positioned according to the standard 10-10 EEG electrode placement. ${ }^{35}$

\subsection{Experiments of Simultaneous EEG-tPBM}

Figure 1(c) shows the experimental design for both sham and active tPBMs. Experiments consisted of a 2-min baseline period (Bl), an 11-min laser stimulation period (T1-T11), and a 3-min recovery period (R1-R3). All 64-channel EEG data were acquired throughout the 16-min duration. Each subject received both active and sham stimulations on the same day, with sham taken first, followed by active stimulation. All subjects were unaware that one of the sessions consisted of sham stimulation. After each experiment, each subject was asked about perceptions of heat from the stimulation.

\subsection{EEG Data Analysis}

We conducted data analysis on 64-channel EEG time series in several steps.

\subsubsection{Pre-processing}

All 64-channel EEG time series were preprocessed using the EEGLAB toolbox on the MATLAB platform. ${ }^{36}$ Each of the raw time series was first bandpass filtered between 0.5 and $70 \mathrm{~Hz}$ and then notch-filtered to remove $60-\mathrm{Hz}$ line noise. To remove artifacts from eye blinks, saccades, and jaw clenches, we performed independent component analysis ${ }^{37}$ via the EEGLAB function "runica." Artifactual components were visually identified and removed from the data. ${ }^{38,39}$ Electrode $\mathrm{Cz}$ was used as the reference for the other 63 EEG channels.

\subsubsection{Selection of frequency bands}

For spatial topographies of EEG power spectral densities, we selected the five commonly used EEG frequency bands; namely, delta $(0.5$ to $4 \mathrm{~Hz})$, theta (4 to $8 \mathrm{~Hz})$, alpha ( 8 to $13 \mathrm{~Hz}$ ), beta (13 to $30 \mathrm{~Hz}$ ), and gamma (30 to $70 \mathrm{~Hz}$ ) frequency bands.

\subsubsection{Baseline quantification}

For each of the aforementioned five frequency bands, the 60-s prelaser (or baseline) EEG power spectral densities were temporally averaged for each EEG channel for both active and sham tPBM group data. Then, we performed a two-sample $t$-test between the EEG baselines during active and sham tPBMs for each of 64 channels. The results showed no statistically significant difference between the baselines of the active and sham group data for each of the five bands.

\subsubsection{Time-resolved, baseline-normalized topography}

For each frequency band, we created time-dependent, 64channel (i.e., $64 \times 1$ ) power density vectors, $P_{\mathrm{tPBM}}$ or $P_{\text {sham }}$, for either tPBM or sham experiment during the stimulation (11 min) and recovery (3 $\mathrm{min})$ period, as well as a corresponding baseline power density vector, $P_{\text {base }}$ (based on the second-minute 

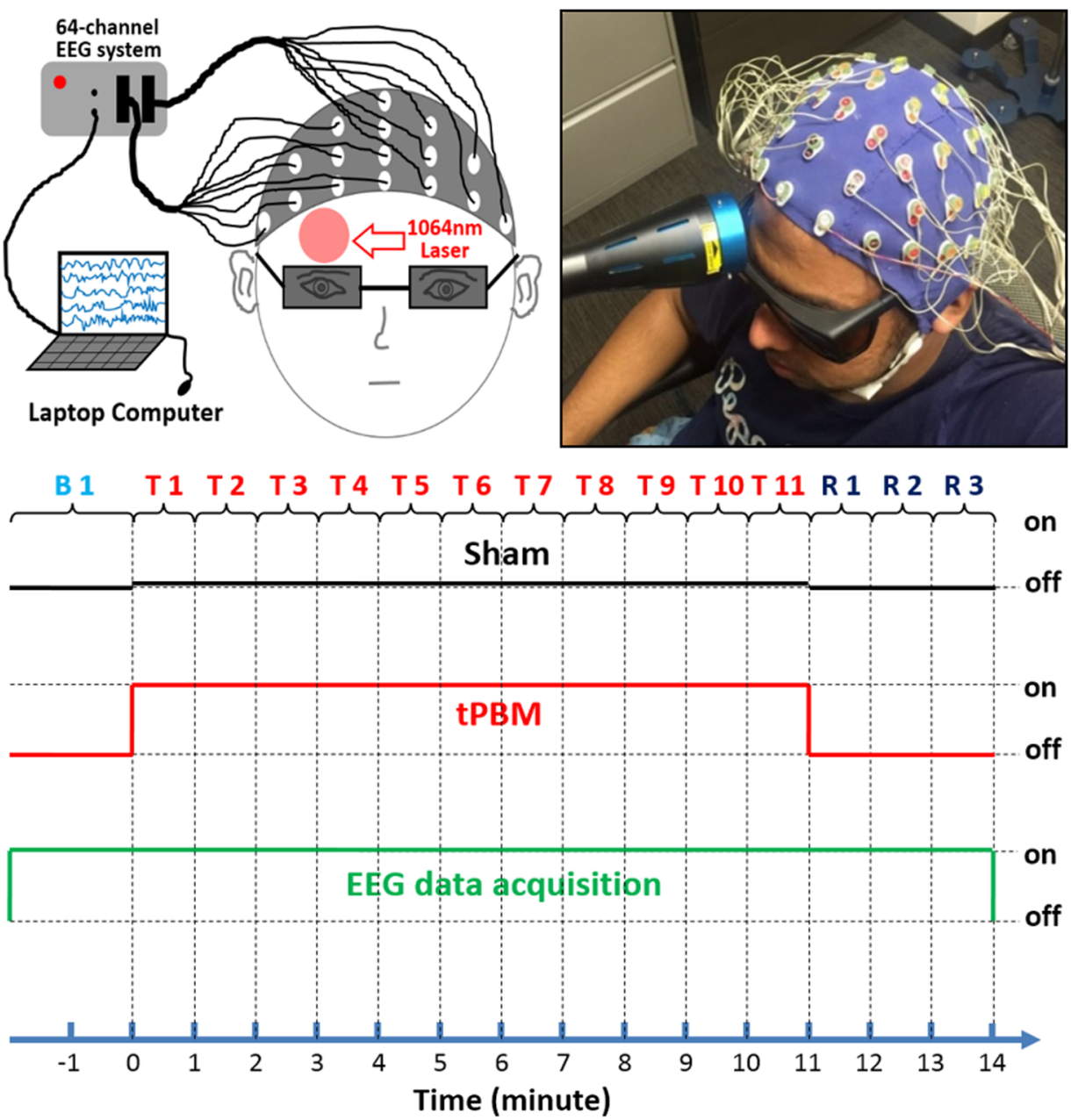

Fig. 1 (a) A highly schematic drawing of the setup, (b) a photo of actual setup of the EEG-tPBM experimental setup, and (c) illustration of the study protocol for EEG-tPBM and EEG-sham experiments.

baseline data). Baseline-normalized, tPBM-induced (or shaminduced) increases in power density were quantified as $\mathrm{n} P_{\mathrm{tPBM}}=P_{\mathrm{tPBM}} / P_{\text {base }}-1$ (or $\left.\mathrm{n} P_{\text {sham }}=P_{\text {sham }} / P_{\text {base }}-1\right)$ for each of the 14 time-dependent periods during and after tPBM (i.e., T1-T11 and R1-R3). This data processing routine is shown in Fig. 2. Next, after close inspection on the magnitudes of time-resolved, baseline-normalized EEG power density for each EEG channel at each frequency band, we excluded three subjects as outliers. The exclusion criterion was that any subject whose laser-induced power alteration (i.e., increase or decrease) in any electrode was equal to or larger than four standard deviations away from the sample/group mean would be excluded as an outlier, regardless of any channel or/and any frequency band. In other words, we calculated the sample/group mean $\left(\mu_{\text {sample }}\right)$ and standard deviation $\left(\sigma_{\text {sample }}\right)$ of the power ratios, for each channel at each frequency, for all the subjects. The ratios that were larger than $\mu_{\text {sample }}+4 \sigma_{\text {sample }}$ or smaller than $\mu_{\text {sample }}-$ $4 \sigma_{\text {sample }}$, were regarded as outliers, and the corresponding participant was removed from further data processing.

\subsubsection{Statistical analysis}

Mean values of $\mathrm{n} P_{\mathrm{tPBM}}$ and $\mathrm{n} P_{\text {sham }}$ over 17 participants at each temporal point were calculated, and then the mean differences with respect to the baseline power density were obtained and presented by two-dimensional topographic maps in temporal sequence (in minutes). Next, we performed two-sample $t$-tests between mean $\mathrm{n} P_{\mathrm{tPBM}}$ and $\mathrm{n} P_{\text {sham }}$ (i.e., comparing active and sham tPBM experiments) in temporal sequence, with a statistical significance level chosen to be $p<0.05$ after Bonferroni correction (see Fig. 2), which is commonly used for multiple comparison to reduce type I error. A total of 14 statistical T-maps were made, for each frequency band, to visually show regions where tPBM induced significant increases in EEG power densities on the human head. Moreover, using another way to present statistically significant results, we calculated time-resolved effect size for the group differences using Cohen's " $d$ " 40 and displayed it on topographic maps, following the similar presentation given in Ref. 41 .

\section{Results}

A sham-controlled within-subjects design with $n=20$ healthy human participants was employed to assess the effect of tPBM on the power of EEG oscillations at five canonical bands. After excluding three subjects whose time-resolved magnitudes of baseline-normalized EEG power density met the exclusion criterion, we reported group effects derived from the remaining 17 participants.

Figure 3(a) shows time-resolved spatial topographies of group-level differences in baseline-normalized EEG power 


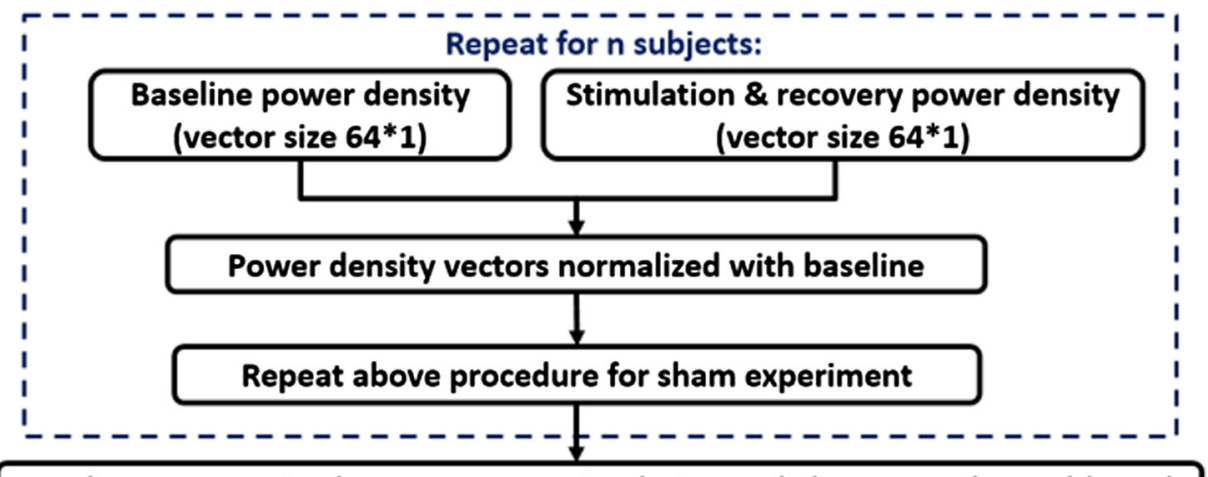

T-value computation between Laser stimulation and Sham at each voxel $(n=17)$

Fig. 2 A flow chart for EEG data analysis to obtain topographic maps of EEG power spectral density differences between active and sham tPBMs, followed by time-dependent the $t$-test for statistical comparison. This process routine was used for each of the five frequency bands.

(a)



(b)

T-map
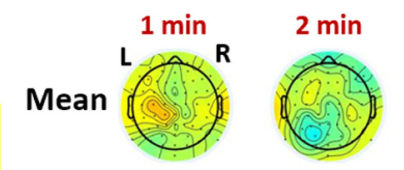

Theta $(4-8 \mathrm{~Hz})$

\section{$T>3$.}
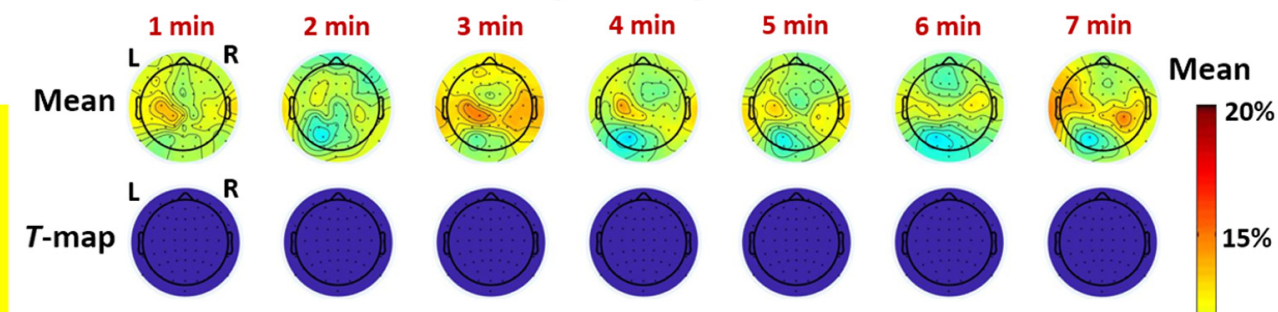

R 1 min

R 2 min

R 3 min

Mean
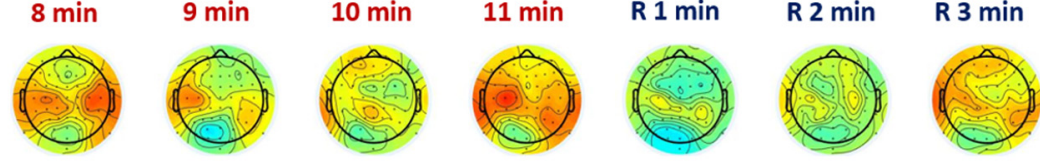

$T$-map


$0 \%$

Fig. 3 Time-resolved topographic maps of group-level differences in baseline-normalized EEG power density at (a) delta and (b) theta bands between $\mathrm{tPBM}$ and sham experiments (i.e., $\mathrm{n} P_{\mathrm{TPBM}}-\mathrm{n} P_{\text {sham }}$ ) and corresponding statistical T-maps, during the $11 \mathrm{~min}$ of tPBM/sham $(\mathrm{T} 1, \mathrm{~T} 2, \ldots \mathrm{T} 11)$ and $3 \mathrm{~min}$ of recovery (R1, R2, and R3) periods. For both (a) and (b) panels, the color bar on the right side represents mean difference in percentage increase of respective power density between IPBM and sham stimulations. The color bar on the left side represents the T-score cutoff for the Bonferroni-corrected $p$-value $(p<0.05$, which is equivalent to $T>3.6$ ) of significant differences in power density between IPBM and sham stimulations. 
density at the delta $(0.5$ to $4 \mathrm{~Hz})$ frequency band between tPBM and sham experiments (i.e., $\mathrm{n} P_{\mathrm{tPBM}}-\mathrm{n} P_{\text {sham }}$ ) and corresponding statistical T-maps, during the $11 \mathrm{~min}$ of $\mathrm{tPBM} / \mathrm{sham}$ (T1, T2, ... T11) and 3 min of recovery (R1, R2, and R3) periods. Figure 3 (b) presents the corresponding topographies for the theta (4 to $8 \mathrm{~Hz}$ ) frequency band. Figure 3(a) does not show obvious increases in mean magnitude of EEG delta power density at any temporal point with respect to the sham-induced results, and Fig. 3(b) seems to reveal mean magnitude increases of tPBM-induced EEG theta power density at several temporal periods (e.g., time $=3,8,11 \mathrm{~min}$ ) compared to the sham results. However, the respective T-maps after Bonferroni correction indicate that the magnitude of laser-induced changes of EEG powers in both delta and theta bands are not statistically significant across any 14 time periods, suggesting that $\mathrm{PBM}$ has no statistical effects on delta and theta bands.

Figure 4(a) illustrates 14 time-resolved (i) spatial topographies of group-level mean differences in baseline-normalized (a)

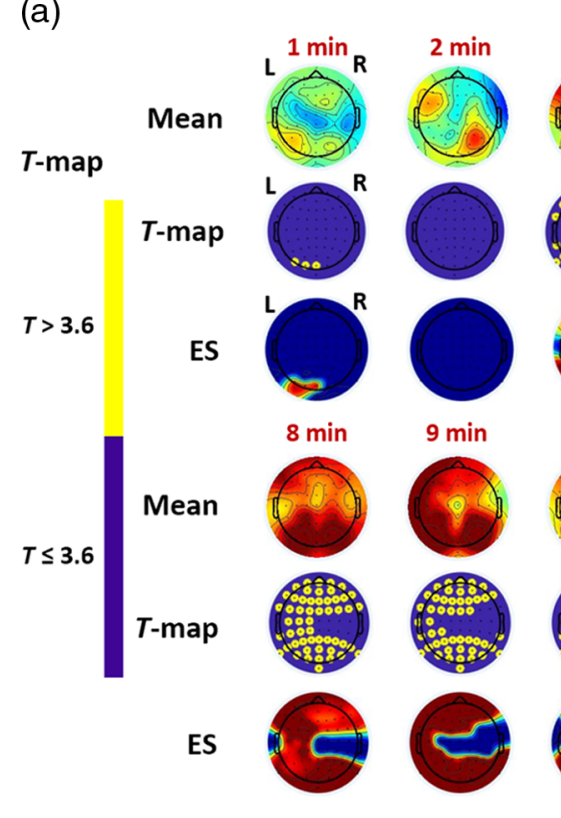

(b)
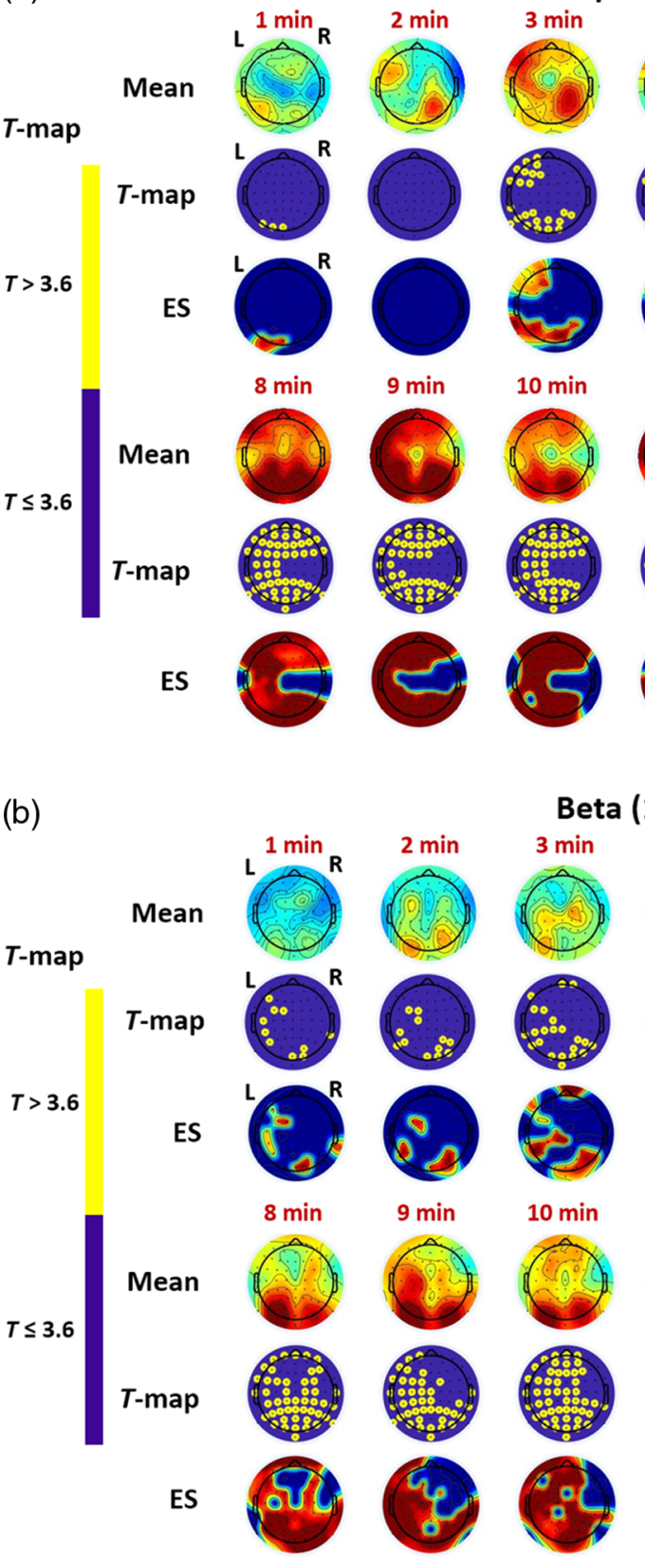

Alpha $(8-13 \mathrm{~Hz})$


$\because$
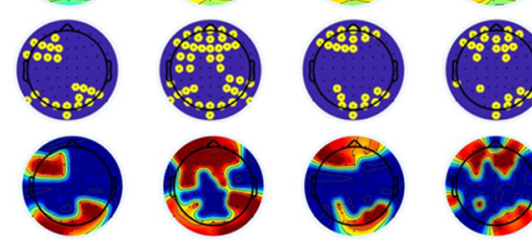

ES Mean
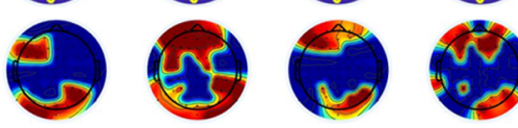

$15 \%$

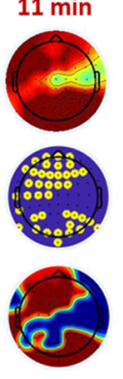

$R 1$ min

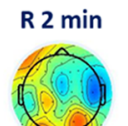

R 3 min


$\mathbf{5 \%}$

$0 \quad 0 \%$

(
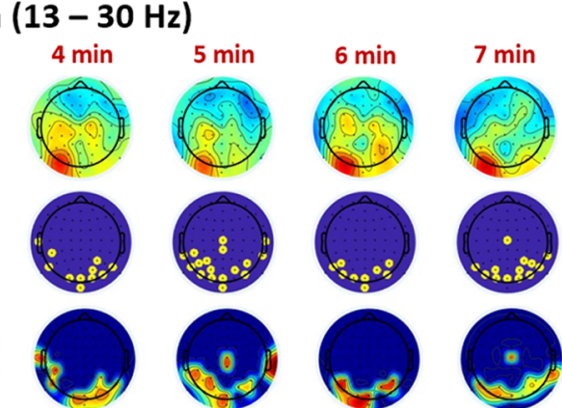

ES Mean
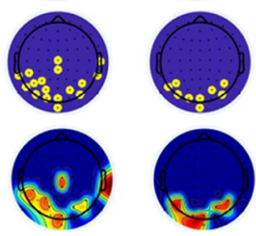

R 1 min

R 2 min
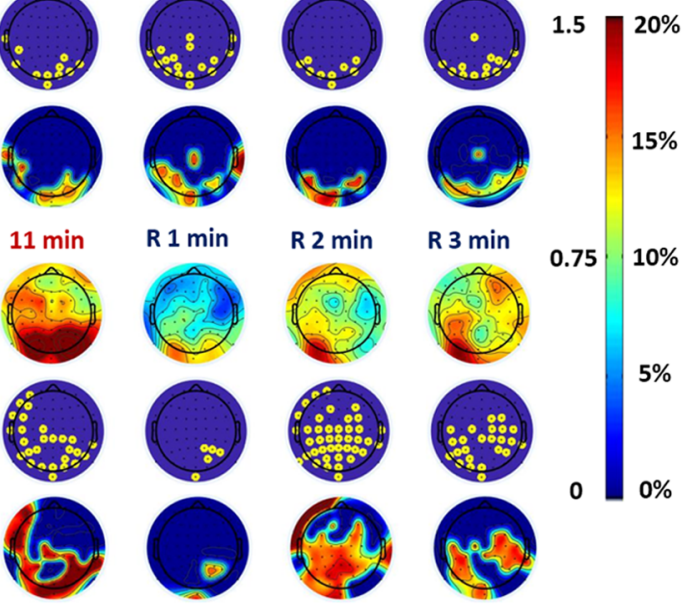

$15 \%$

Fig. 4 Time-resolved topographic maps of group-level differences in baseline-normalized EEG power density at (a) alpha and (b) beta bands between tPBM and sham experiments (i.e., $\mathrm{n} P_{\mathrm{TPBM}}-\mathrm{n} P_{\text {sham }}$ ), corresponding statistical T-maps, and respective effect size maps, during the $11 \mathrm{~min}$ of $\mathrm{tPBM} / \mathrm{sham}$ $(\mathrm{T} 1, \mathrm{~T} 2, \ldots \mathrm{T} 11)$ and 3 min of recovery $(\mathrm{R} 1, \mathrm{R} 2$, and R3) periods. For both (a) and (b) panels, the color bar on the right side represents both the effect size (ES) and the mean difference in percentage increase of respective power density between tPBM and sham stimulations. The color bar on the left side represents the T-score cutoff for the Bonferroni-corrected $p$-value $(p<0.05$, which is equivalent to $T>3.6)$ of significant differences in power density between $\mathrm{PBM}$ and sham stimulations. 
EEG power density at the alpha (8 to $13 \mathrm{~Hz}$ ) frequency band between $\mathrm{tPBM}$ and sham experiments, (ii) corresponding statistical T-maps, and (iii) respective effect-size topographic maps, during and after $\mathrm{TPBM}$. This figure shows that alpha power increases in mean magnitude after tPBM onset and remains elevated over bilateral anterior and posterior regions even after stimulation. Statistical T-maps after Bonferroni correction show that stimulation of the right forehead by tPBM increases the alpha oscillation powers significantly over many bilateral regions, anteriorly and posteriorly, on the human head throughout the stimulation time, particularly 8 to $11 \mathrm{~min}$ after the stimulation onset. This observation is supported by the effect-size maps, which demonstrate that tPBM causes very large effects in alpha power as compared to the sham control in the above mentioned regions and temporal periods, given that $|d|<0.2$ - small effect; $0.2<|d|<0.8$ - medium effect; $|d|>0.8$ - large effect; $|d|>1.2$ - very large effect. ${ }^{41}$

Figure 4(b) illustrates 14 time-resolved (i) spatial topographies of group-level mean differences in baseline-normalized EEG power density at the beta $(13$ to $30 \mathrm{~Hz})$ frequency band between $\mathrm{TPBM}$ and sham experiments, (ii) corresponding statistical T-maps, and (iii) respective effect-size topographic maps, during and after tPBM. Increases of beta-band power are also observed, occurring mainly over the posterior electrodes 4 to $7 \mathrm{~min}$ after stimulation onset. Similar to the alpha band, tPBM-induced beta oscillation powers are elevated significantly across most of the scalp (both anterior and posterior) 8 to $11 \mathrm{~min}$ after tPBM onset. The statistical T-maps shown in Fig. 4(b) also reveal statistically significant differences in beta power density between active and sham tPBMs during the recovery period (i.e., during R1, R2, and R3) in several scalp regions. This observation is further confirmed by the respective effect-size maps, illustrating that $\mathrm{PBM}$ causes very large effects in beta power as compared to the sham control in the above-mentioned regions and temporal periods, given that $|d|<0.2-$ small effect; $0.2<|d|<0.8$ - medium effect; $|d|>0.8$ - large effect; $|d|>$ 1.2 - very large effect. ${ }^{41}$

Regarding electrophysiological responses of the brain to tPBM at the gamma (30 to $70 \mathrm{~Hz}$ ) frequency band, Fig. 5 seems to show some mean magnitude changes anteriorly and posteriorly, particularly during 8 to $11 \mathrm{~min}$ after the stimulation onset. However, the Bonferroni-corrected statistical analysis as shown by corresponding T-maps in Fig. 5 indicates that the laserinduced changes of EEG gamma powers are not statistically significant between the laser and sham stimulations across any 14 time periods.

\section{Discussion}

The findings have provided the first detailed demonstration that $\mathrm{PBM}$ significantly increased the strength of electrophysiological oscillations at the alpha and beta frequencies in different brain regions across the human scalp. Following a shamcontrolled within-subjects design, we applied tPBM to the right forehead of 20 human subjects while recording the scalp EEG to investigate time-resolved, large-scale, electrophysiological changes across the entire scalp in response to tPBM. Our multistep analysis revealed several novel and intriguing findings, answered our research questions, proved our research hypothesis, and showed several limitations of this study.

\section{1 tPBM Modulates Alpha and Beta Power}

Figure 4 illustrates that the 11-min tPBM delivered to the right forehead of 20 human subjects (three outliers excluded) results in up to $20 \%$ spatially broad increases of human alpha and beta band powers. Figure 4(a) reveals that, in the alpha band, the scalp EEG oscillation powers increased progressively after the laser stimulation was initiated relative to the sham condition. Furthermore, the strongest increases in alpha oscillation power appeared during 8 to 11 min with a dominant front-to-back



Fig. 5 Time-resolved topographic maps of group-level differences in baseline-normalized EEG power density at gamma band between IPBM and sham experiments and corresponding statistical T-maps, during the $11 \mathrm{~min}$ of $\mathrm{PBBM} / \mathrm{sham}$ and $3 \mathrm{~min}$ of recovery periods. The color bar on the right side represents mean difference in percentage increase of gamma power density between IPBM and sham stimulations. The color bar on the left represents the $t$-score cutoff for the Bonferroni-corrected $p$-value $(p<0.05$, which is equivalent to $T>3.6$ ) of significant differences in power density between tPBM and sham stimulations. 
pattern bilaterally. Figure 4(b) shows a similar trend of rhythm power increases for the beta band, particularly still remaining the dominant front-to-back pattern during the later period of tPBM (i.e., during 8 to $11 \mathrm{~min}$ ).

Together the figures reveal several key features: (1) laserinduced increases of EEG power density were frequencydependent since the statistically significant increases occurred only at the alpha and beta bands, with very strong effects (maximum effect size $\cong 1.5$ ); (2) laser-induced alterations were cumulatively time-dependent since the changes took place on a time scale of minutes for both alpha and beta bands, and they were largest during 8 to $11 \mathrm{~min}$ after laser onset; and (3) the alterations were location-dependent, based on the observations that significant increases in power density appeared to be frontto-back on the scalp 3 min after stimulation onset for the alpha and beta bands, with increases remaining front-to-back for the alpha band and for the beta band after 7 min of the stimulation onset.

It is commonly regarded that an increase in alpha power alone implies a less attentive state and less arousal. It might be argued that the increase of alpha power seen in Fig. 4(a) could result from the subjects' sleepiness as they sat in the experiment for a while. If subjects get sleepy, alpha increases but not both alpha and beta as produced by the laser as compared to the sham with the same temporal period. Therefore, the tPBM effect is not the same as simply lowering arousal level. This difference may be important for the cognitive benefit produced by the tPBM. It is also reported that alpha and beta rhythms of brain oscillations result from synchronous electrical activity of thalamocortical neurons, with alpha more characteristic of quiet awake states and beta of alert states. ${ }^{42,43}$ More specifically, alpha waves are robustly modulated during cognitive processes and play an important role in the integration and communication among different brain rhythms during brain activity. ${ }^{44}$ Beta oscillations are also relevant to cognitive processing but to a lesser degree than alpha waves. ${ }^{45,46}$ Our results reveal that tPBM is able to create large effects on not only alpha but also beta oscillations. All of these observations lead us to speculate that the laserinduced large effect in both alpha and beta powers may be a mechanistic link to tPBM-induced improvement and enhancement of human cognition that have been repeatedly reported in sham-controlled studies by Gonzalez-Lima et al. and others in recent years. ${ }^{13-16,30}$ As supporting evidence for this speculation, many studies in neurofeedback research have observed improvement of cognitive performance while the upper alpha amplitude increased ${ }^{47}$ by neurofeedback training. ${ }^{47-51}$

We have also noticed that prefrontal tPBM has created a dominant front-to-back pattern in both alpha and beta rhythm powers, with a few minutes delay in the beta power increase. Since there is no prior literature on such observation, one possible speculation is to attribute this pattern to tPBM-induced enhancement in large-scale brain circuits in one or more prefrontal-driven brain networks, such as the frontoparietal central executive network and the default mode network (DMN) (see Sec. 4.2 next for more details). The former network is known for initiation and modulation of cognitive control. ${ }^{52}$ If prefrontal tPBM is proven to be able to modulate this network, it would reveal an important electrophysiological mechanism of action of tPBM at the brain circuit level and thus lead to a better understanding of tPBM-based interventions for both neuroscience research and clinical applications. Our speculation needs to be investigated and tested in future studies.

\section{2 tPBM Holds Potential for Photobiomodulation of the Human Default Mode Network}

The DMN is a network of brain areas activated during the resting state; the DMN becomes suppressed once certain tasks are involved. ${ }^{53}$ Malfunction and disconnection of the DMN have been related to the cognitive dysfunctions seen in many psychiatric and neurological conditions, such as depression, anxiety, and Alzheimer's disease. ${ }^{53-55}$ The abnormal connectivity of the DMN in those conditions makes the brain unable to stay in a normal resting state. ${ }^{53}$ Increases of EEG power density in the anterior frontal regions indicate that $\mathrm{PBM}$ may have the potential or ability to strengthen and stimulate neural functions of the anterior DMN. In particular, the T-maps of EEG alpha power during 8 to $11 \mathrm{~min}$ [Fig. 4(a)] illustrate that the scalp area covering (entirely or partially) the anterior DMN is significantly modulated by tPBM. We note, however, that brain imaging modalities with higher spatial resolution than that of EEG will be needed to pinpoint the areas modulated by tPBM. The modulatory potential of $\mathrm{tPBM}$ on the DMN suggests a promising prospect for $\mathrm{PBM}$ to promote DMN functions in healthy people and perhaps reverse DMN changes found in various psychiatric and neurological conditions such as depression, anxiety, and Alzheimer's disease.

\subsection{Continuous-Wave versus Pulsed tPBM}

The stimulation light used in our current ${ }^{17,18}$ and previous ${ }^{13-15}$ studies was CW laser, and most light sources employed in other tPBM investigations were also nonpulsed lasers or LEDs. To date, it is still unclear whether CW PBM is better or worse than pulsed PBM for different uses (e.g., physical therapy, chiropractic, pain reduction, sports medicine), ${ }^{56}$ and very limited reports with pulsed $\mathrm{TPBM}$ can be found. For example, a human study with a $10-\mathrm{Hz}$ pulse frequency was recently published, ${ }^{57}$ and several preclinical studies explored treatment outcomes of pulsed tPBM on different types of neurological conditions with different pulse frequencies. ${ }^{58-62}$ Table 4 lists corresponding pulsed frequencies, studied species, and related references. In principle, one advantage of CW tPBM is to deliver more optical energy/ fluence, and thus more dosage in a given time, but one disadvantage is to possibly create more heat on the skin surface. The answer to the question of whether CW tPBM is better or worse with respect to pulsed $\mathrm{PBM}$ for affecting EEG rhythms or other applications remains to be further investigated for the entire tPBM field.

Table 4 Studies using pulsed tPBM.

\begin{tabular}{lcc}
\hline Reference & Species & $\begin{array}{c}\text { Pulsed } \\
\text { frequency }(\mathrm{Hz})\end{array}$ \\
\hline Saltmarche et al. ${ }^{57}$ & Human & 10 \\
Lapchak et al. $^{58}$ & Rabbits & $1 \mathrm{k}$ \\
Oron et al. ${ }^{59}$ & Rat & $10 \mathrm{k}$ \\
Zhang et al. $^{60}$ & Rat & 70 \\
Blivet et al. & & \\
Oron et al. ${ }^{62}$ & Mice & 10 \\
\hline
\end{tabular}




\subsection{Limitations of the Study and Future Work}

Since this was the first sham-controlled, time-resolved topographic study of EEG-tPBM in healthy humans, it had a few limitations that could be overcome in future investigations. First, to prevent the subjects from falling asleep during the EEG measurements, the subjects kept their eyes open during the measurements while wearing safety goggles with a layer of light-absorbing sheet over the edges for eye protection. However, in order to investigate tPBM-induced effects thoroughly on alpha oscillations, it might be appropriate to conduct future experiments with the eyes closed, enabling detection of stronger alpha activity. Second, while the EEG data in this study were acquired using a conventional 10-10 system of scalp electrode placement, the EEG cap was positioned more posteriorly than the regular setup in order to have an adequate space for a 4-cm-diameter laser stimulation site [see Fig. 1(b)]. The shift of the cap was $\sim 2 \mathrm{~cm}$ up along the anterior-posterior midline (or the nasion to inion line), which would cause inconsistency between the conventional 10-10 system electrode sites versus the actual ones used in this study. This mismatch would prevent us from identifying individual scalp locations/regions that have significant EEG power responses to tPBM with high accuracy. A solution to this limitation may be to coregister the actual EEG electrodes with the conventional electrode placement using a three-dimensional digitizer to measure several key anatomical landmarks, followed by coordinate transformation. Third, we speculated that the tPBM-induced increase in both alpha and beta power density may be a mechanistic link to tPBM-induced improvement and enhancement of human cognition that have been reported in sham-controlled studies in recent years. ${ }^{13-16,30}$ This speculation needs to be further investigated and confirmed by combining EEG-tPBM measurements with tPBM-enhanced human cognition tests in the future. Last, we cannot rule out that heating did not play a role in the electrophysiological changes observed here. Cortical temperature is known to change with neural activation by $\sim 0.2-\mathrm{deg}$ Celsius. ${ }^{63}$ It is possible that the reverse effect-exogenous heat alters neural activity - could have contributed to the observed effects. On the other hand, we have previously shown that thermal stimulation of the forehead does not lead to the increased cortical CCO or hemodynamic changes with $\mathrm{PPBM}^{64}$ In the future, a similar thermal stimulation study of EEG and computational simulations may be possible approaches to quantify or estimate tPBM-induced versus thermal effects on the EEG and thus be able to disambiguate the effects of photobiomodulation and tissue heating.

\section{Conclusion}

This is the first time-resolved topography report to demonstrate that tPBM with 1064-nm laser increases the power of brain alpha and beta rhythm oscillations significantly and broadly across the scalp, in particular across bilateral anterior and posterior sites. The scalp EEG was utilized to measure shamcontrolled, human electrophysiological rhythms before, during, and after 11-min $\mathrm{TPBM}$ delivered to the right forehead of a cohort of 20 normal human subjects. By computing oscillatory power in a time-resolved fashion, we have found that $\mathrm{TBB}$ produced time-varying statistically significant increases in EEG power in both the alpha and the beta bands. The electrodes of significant effects cover the scalp regions with a front-to-back pattern, peaking $8 \mathrm{~min}$ after stimulation onset. Thus, tPBM modulated the synchronization of neural activity in the alpha and beta frequency ranges. Together with previous studies, this EEG study points to 1064-nm infrared light as a form of transcranial brain stimulation or neuromodulation by targeting cellular energy metabolism and associated changes in hemodynamics and electrophysiological activity of the brain.

\section{Disclosures}

No conflicts of interest, financial or otherwise, are declared by all the authors.

\section{Acknowledgments}

This work was supported in part by the National Institute of Mental Health/National Institutes of Health under the BRAIN Initiative (RF1MH114285). We also acknowledge the support in part from the STARS program by the University of Texas System. FGL was supported in part by the Oskar Fischer Project Fund.

\section{References}

1. J. T. Eells et al., "Mitochondrial signal transduction in accelerated wound and retinal healing by near-infrared light therapy," Mitochondrion 4(5-6), 559-567 (2004).

2. M. T. Wong-Riley et al., "Photobiomodulation directly benefits primary neurons functionally inactivated by toxins: role of cytochrome c oxidase," J. Biol. Chem. 280(6), 4761-4771 (2005).

3. M. A. Naeser et al., "Improved cognitive function after transcranial, light-emitting diode treatments in chronic, traumatic brain injury: two case reports," Photomed. Laser Surg. 29(5), 351-358 (2011).

4. J. J. Anders et al., "In vitro and in vivo optimization of infrared laser treatment for injured peripheral nerves," Lasers Surg. Med. 46(1), 34-45 (2014).

5. R. T. Chow, "Low-level laser therapy of pain: clinical applications," in Handbook of Low-Level Laser Therapy, M. R. Hamblin, M. V. Sousa, and T. Agrawal, Eds., pp. 641-684, Pan Stanford Publishing, Singapore (2017).

6. D. Hawkins, N. Houreld, and H. Abrahamse, "Low level laser therapy (LLLT) as an effective therapeutic modality for delayed wound healing," Ann. N. Y. Acad. Sci. 1056, 486-493 (2005).

7. C. E. Tedford et al., "Quantitative analysis of transcranial and intraparenchymal light penetration in human cadaver brain tissue," Lasers Surg. Med. 47(5), 466-466 (2015).

8. J. C. Rojas and F. Gonzalez-Lima, "Neurological and psychological applications of transcranial lasers and LEDs," Biochem. Pharmacol. 86(4), 447-457 (2013).

9. Y. Lampl, "Laser treatment for stroke," Expert Rev. Neurother. 7(8), 961-965 (2007).

10. M. A. Naeser and M. R. Hamblin, "Potential for transcranial laser or LED therapy to treat stroke, traumatic brain injury, and neurodegenerative disease," Photomed. Laser Surg. 29(7), 443-446 (2011).

11. P. Cassano et al., "Review of transcranial photobiomodulation for major depressive disorder: targeting brain metabolism, inflammation, oxidative stress, and neurogenesis," Neurophotonics 3(3), 031404 (2016).

12. M. H. Berman et al., "Photobiomodulation with near infrared light helmet in a pilot, placebo controlled clinical trial in dementia patients testing memory and cognition," J. Neurol. Neurosci. 8(1), 176 (2017).

13. D. W. Barrett and F. Gonzalez-Lima, "Transcranial infrared laser stimulation produces beneficial cognitive and emotional effects in humans," Neuroscience 230, 13-23 (2013).

14. N. J. Blanco, C. L. Saucedo, and F. Gonzalez-Lima, "Transcranial infrared laser stimulation improves rule-based, but not information-integration, category learning in humans," Neurobiol. Learn. Mem. 139, 69-75 (2017).

15. N. J. Blanco, W. T. Maddox, and F. Gonzalez-Lima, "Improving executive function using transcranial infrared laser stimulation," J. Neuropsychol. 11(1), 14-25 (2017).

16. E. Vargas et al., "Beneficial neurocognitive effects of transcranial laser in older adults," Lasers Med. Sci. 32(5), 1153-1162 (2017). 
17. F. Tian et al., "Transcranial laser stimulation improves human cerebral oxygenation," Lasers Surg. Med. 48(4), 343-349 (2016).

18. X. Wang et al., "Up-regulation of cerebral cytochrome-c-oxidase and hemodynamics by transcranial infrared laser stimulation: a broadband near-infrared spectroscopy study," J. Cereb. Blood Flow Metab. 37(12), 3789-3802 (2017).

19. X. Wang et al., "Interplay between up-regulation of cytochrome-coxidase and hemoglobin oxygenation induced by near-infrared laser," Sci. Rep. 6, 30540 (2016)

20. J. C. Rojas and F. Gonzalez-Lima, "Low-level light therapy of the eye and brain," Eye Brain 3, 49-67 (2011).

21. M. R. Hamblin, "Introduction to experimental and clinical studies using low-level laser (light) therapy (LLLT)," Lasers Surg. Med. 42(6), 447-449 (2010).

22. M. T. Wong-Riley, "Cytochrome oxidase: an endogenous metabolic marker for neuronal activity," Trends Neurosci. 12(3), 94-101 (1989).

23. X. Wang et al., "Proceedings \#18. Transcranial infrared brain stimulation modulates EEG alpha power," Brain Stimul. 10(4), e67-e69 (2017).

24. M. Bortoletto et al., "The contribution of TMS-EEG coregistration in the exploration of the human cortical connectome," Neurosci. Biobehav. Rev. 49, 114-124 (2015).

25. P. Schestatsky, L. Morales-Quezada, and F. Fregni, "Simultaneous EEG monitoring during transcranial direct current stimulation," J. Visualized Exp. 76, e50426 (2013).

26. M. Hallett, "Transcranial magnetic stimulation: a primer," Neuron 55(2), 187-199 (2007).

27. S. Palva and J. M. Palva, "New vistas for alpha-frequency band oscillations," Trends Neurosci. 30(4), 150-158 (2007).

28. S. Palva and J. M. Palva, "Functional roles of alpha-band phase synchronization in local and large-scale cortical networks," Front. Psychol. 2, 204 (2011).

29. M. Tanaka et al., "Effect of mental fatigue on the central nervous system: an electroencephalography study," Behav. Brain Funct. 8, 48 (2012).

30. J. Hwang, D. M. Castelli, and F. Gonzalez-Lima, "Cognitive enhancement by transcranial laser stimulation and acute aerobic exercise," Lasers Med. Sci. 31(6), 1151-1160 (2016).

31. P. Jenkins and J. D. Carroll, "How to report low-level laser therapy (LLLT)/photomedicine dose and beam parameters in clinical and laboratory studies," Photomed. Laser Surg. 29(12), 785-787 (2011).

32. P. A. Lapchak et al., "Transcranial near-infrared laser transmission (NILT) profiles $(800 \mathrm{~nm})$ : systematic comparison in four common research species," PLoS One 10(6), e0127580 (2015).

33. A. Pitzschke et al., "Red and NIR light dosimetry in the human deep brain," Phys. Med. Biol. 60(7), 2921-2937 (2015).

34. J. R. Jagdeo et al., "Transcranial red and near infrared light transmission in a cadaveric model," PLoS One 7(10), e47460 (2012).

35. V. Jurcak, D. Tsuzuki, and I. Dan, "10/20, 10/10, and 10/5 systems revisited: their validity as relative head-surface-based positioning systems," Neuroimage 34(4), 1600-1611 (2007).

36. A. Delorme and S. Makeig, "EEGLAB: an open source toolbox for analysis of single-trial EEG dynamics including independent component analysis," J. Neurosci. Methods 134(1), 9-21 (2004).

37. A. Hyvarinen and E. Oja, "Simple neuron models for independent component analysis," Int. J. Neural Syst. 7(6), 671-687 (1996).

38. T. P. Jung et al., "Removing electroencephalographic artifacts by blind source separation," Psychophysiology 37(2), 163-178 (2000).

39. D. Lehmann and W. Skrandies, "Reference-free identification of components of checkerboard-evoked multichannel potential fields," Electroencephalogr. Clin. Neurophysiol. 48(6), 609-621 (1980).

40. J. Cohen, The Statistical Power Analysis for the Behavioural Sciences, 2nd edn., Lawrence Erlbaum Associates, Hillsdale, New Jersey (1988).

41. E. Neto et al., "EEG spectral features discriminate between Alzheimer's and vascular dementia," Front. Neurol. 6, 25 (2015).

42. S. Vijayan and N. J. Kopell, "Thalamic model of awake alpha oscillations and implications for stimulus processing," Proc. Natl. Acad. Sci. U.S.A. 109(45), 18553-18558 (2012).

43. W. J. Ray and H. W. Cole, "EEG alpha activity reflects attentional demands, and beta activity reflects emotional and cognitive processes," Science 228(4700), 750-752 (1985).

44. W. Klimesch, "Alpha-band oscillations, attention, and controlled access to stored information," Trends Cognit. Sci. 16(12), 606-617 (2012).
45. I. Timofeev et al., "Neuronal synchronization and thalamocortical rhythms in sleep, wake and epilepsy," in Jasper's Basic Mechanisms of the Epilepsies, J. L. Noebels et al., Eds., 4th edn., National Center for Biotechnology Information, Bethesda, Maryland, https:// www.ncbi.nlm.nih.gov/books/NBK98144/ (2012).

46. B. Guntekin et al., "Beta oscillatory responses in healthy subjects and subjects with mild cognitive impairment," Neuroimage Clin. 3, 39-46 (2013).

47. B. Zoefel, R. J. Huster, and C. S. Herrmann, "Neurofeedback training of the upper alpha frequency band in EEG improves cognitive performance," Neuroimage 54(2), 1427-1431 (2011).

48. F. Wan et al., "Resting alpha activity predicts learning ability in alpha neurofeedback," Front. Hum. Neurosci. 8, 500 (2014).

49. C. Escolano, M. Aguilar, and J. Minguez, "EEG-based upper alpha neurofeedback training improves working memory performance," in Annu. Int. Conf. IEEE Eng. Med. Biol. Soc., pp. 2327-2330 (2011).

50. J. Rogala et al., "The Do's and Don'ts of neurofeedback training: a review of the controlled studies using healthy adults," Front. Hum. Neurosci. 10, 301 (2016).

51. A. Mottaz et al., "Neurofeedback training of alpha-band coherence enhances motor performance," Clin. Neurophysiol. 126(9), 1754-1760 (2015).

52. T. P. Zanto and A. Gazzaley, "Fronto-parietal network: flexible hub of cognitive control," Trends Cognit. Sci. 17(12), 602-603 (2013).

53. R. L. Buckner, J. R. Andrews-Hanna, and D. L. Schacter, "The brain's default network: anatomy, function, and relevance to disease," Ann. $N$. Y. Acad. Sci. 1124, 1-38 (2008).

54. M. D. Greicius et al., "Default-mode network activity distinguishes Alzheimer's disease from healthy aging: evidence from functional MRI," Proc. Natl. Acad. Sci. U.S.A. 101(13), 4637-4642 (2004).

55. K. Mevel et al., "The default mode network in healthy aging and Alzheimer's disease," Int. J. Alzheimers Dis. 2011, 535816 (2011).

56. J. T. Hashmi et al., "Effect of pulsing in low-level light therapy," Lasers Surg. Med. 42(6), 450-466 (2010).

57. A. E. Saltmarche et al., "Significant improvement in cognition in mild to moderately severe dementia cases treated with transcranial plus intranasal photobiomodulation: case series report," Photomed. Laser Surg. 35(8), 432-441 (2017).

58. P. A. Lapchak, J. Wei, and J. A. Zivin, "Transcranial infrared laser therapy improves clinical rating scores after embolic strokes in rabbits," Stroke 35(8), 1985-1988 (2004).

59. A. Oron et al., "Low-level laser therapy applied transcranially to rats after induction of stroke significantly reduces long-term neurological deficits," Stroke 37(10), 2620-2624 (2006).

60. L. Zhang et al., "Quantitative measurement of motor and somatosensory impairments after mild (30 $\mathrm{min})$ and severe $(2 \mathrm{~h})$ transient middle cerebral artery occlusion in rats," J. Neurol. Sci. 174(2), 141-146 (2000).

61. G. Blivet et al., "Neuroprotective effect of a new photobiomodulation technique against $\mathrm{A} \beta_{25-35}$ peptide-induced toxicity in mice: novel hypothesis for therapeutic approach of Alzheimer's disease suggested," Alzheimers Dementia 4, 54-63 (2018).

62. A. Oron et al., "Near infrared transcranial laser therapy applied at various modes to mice following traumatic brain injury significantly reduces long-term neurological deficits," J. Neurotrauma 29(2), 401-407 (2012).

63. D. A. Yablonskiy, J. J. Ackerman, and M. E. Raichle, "Coupling between changes in human brain temperature and oxidative metabolism during prolonged visual stimulation," Proc. Natl. Acad. Sci. U.S.A. 97(13), 7603-7608 (2000).

64. X. Wang et al., "Impact of heat on metabolic and hemodynamic changes in transcranial infrared laser stimulation measured by broadband nearinfrared spectroscopy," Neurophotonics 5(1), 011004 (2018).

Xinlong Wang received his BS degree in applied physics from Beijing University of Technology, China. He received his PhD through the BS to PhD program at the University of Texas (UT) at Arlington, Texas, where he received the "Excellent Graduates" award. Currently, he is a postdoctoral fellow for a joint project among UT Arlington, UT Austin, and UT Southwestern Medical Center at Dallas, Texas, in the area of transcranial infrared photobiomodulation with multimode imaging and its applications.

Jacek P. Dmochowski is an assistant professor of biomedical engineering at the City College of New York. He received his PhD in 
telecommunications from the Institut National de la Recherche Scientifique, Montreal, Canada, for which he was awarded the Canadian Governor General's Academic Gold Medal. His present research is focused on developing new, noninvasive techniques for sensing and modulating brain activity.

Li Zeng received her BS degree in precision instruments and MS degree in optical engineering from Tsinghua University, China, followed by her MS degree in statistics and her PhD in industrial engineering from University of Wisconsin-Madison, Wisconsin. Currently, she is an assistant professor in the Industrial and Systems Engineering Department at Texas A\&M University. Her research interests are quality engineering and applied statistics in complex manufacturing and healthcare systems. She is a member of INFORMS and IISE.

Elisa Kallioniemi received her BS and MS degrees in biomedical engineering from Aalto University, Finland, followed by her PhD in medical physics from University of Eastern Finland. Currently, she is a postdoctoral researcher at the Department of Psychiatry, UT Southwestern Medical Center, Dallas, Texas. She is a neuromodulation scientist with expertise in transcranial magnetic stimulation and neurophysiology. She is interested in understanding the neural mechanisms and effects of noninvasive neuromodulation and using neuromodulation to study brain function.

Mustafa Husain is a professor of psychiatry, neurology, and internal medicine and the director of the Neuromodulation Research and
Therapeutic Program at UT Southwestern Medical Center. Over the past 25 years, he has explored the intricacies of brain neural connections using different forms of neuromodulation techniques. He has been involved in pioneering the development of magnetic seizure therapy. His broad expertise makes him a leader in research and development in neuromodulation and therapeutics.

F. Gonzalez-Lima is a George I. Sanchez Centennial professor at the University of Texas at Austin, Texas, and is a leading researcher on brain energy metabolism, memory, and neurobehavioral disorders. He received his BS (biology) and BA (psychology) degrees from Tulane University, New Orleans. He received his PhD (anatomy and neurobiology) from the University of Puerto Rico School of Medicine and completed a postdoctoral Humboldt Fellowship (neuroscience) at the Technical University Darmstadt, Germany. He was elected as a fellow of the International Behavioral Neuroscience Society and winner of the 2015 Distinguished Texas Scientist Award. His research has been funded for $>30$ years and has contributed $>300$ scientific publications.

Hanli Liu received her MS and PhD degrees in physics from Wake Forest University, North Carolina, followed by postdoctoral training at the University of Pennsylvania. She is a full professor of bioengineering and Distinguished University Professor at the University of Texas at Arlington. She is also a fellow of the American Institute for Medical and Biological Engineering. Her expertise lies in the field of near-infrared spectroscopy of tissues, functional optical brain imaging, transcranial photobiomodulation, and their clinical applications. 